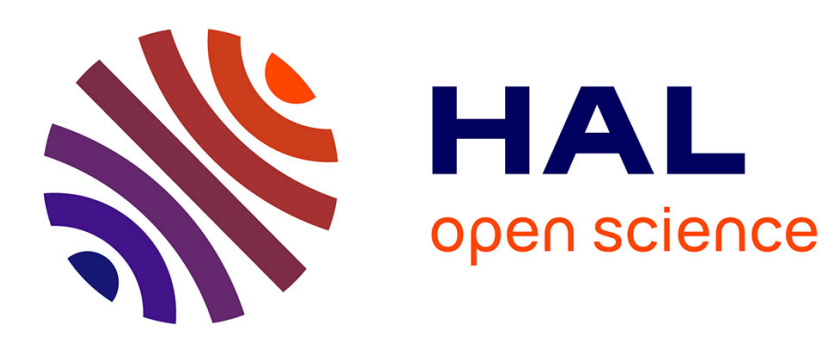

\title{
Mobiliser la souplesse temporelle du client, une stratégie en expansion dans les services
}

Agnès Durrande-Moreau, Jean-Luc Giannelloni

\section{To cite this version:}

Agnès Durrande-Moreau, Jean-Luc Giannelloni. Mobiliser la souplesse temporelle du client, une stratégie en expansion dans les services. Décisions Marketing, 2012, 65, pp.71-76. hal-00951244v2

HAL Id: hal-00951244

https://hal.science/hal-00951244v2

Submitted on 12 Oct 2017

HAL is a multi-disciplinary open access archive for the deposit and dissemination of scientific research documents, whether they are published or not. The documents may come from teaching and research institutions in France or abroad, or from public or private research centers.
L'archive ouverte pluridisciplinaire HAL, est destinée au dépôt et à la diffusion de documents scientifiques de niveau recherche, publiés ou non, émanant des établissements d'enseignement et de recherche français ou étrangers, des laboratoires publics ou privés. 


\title{
MOBILISER LA SOUPLESSE TEMPORELLE DU CLENT Une stratégie en expansion dans les services
}

\author{
AGNÈS DURRANDE-MOREAU ET JEAN-LUC GIANNELLONI
}

Agnès DURRANDE-MOREAU, Maître de conférences HDR, Université de Savoie (IREGE).

Contact : durrande@univ-savoie.fr

Jean-Luc GIANNELLONI, Professeur des Universités, IAE de Grenoble Université Pierre Mendès-France (CERAG UMR 5820)

Contact : jean-luc.giannelloni@iae-grenoble.fr

François $H$. Courvoisier est responsable de la rubrique "stratégies » de la revue Décisions Marketing. Tout auteur souhaitant contribuer à la rubrique peut lui proposer un texte d'environ 2000 à 4300 mots analysant un concept de stratégie marketing et les débats dont il fait l'objet, en l'illustrant d'un ou de plusieurs exemples commentés. Les propositions sont à envoyer à francois.courvoisier@he-arc.ch (chefde rubrique) et au secrétaire de rédaction (decisionsmarketing.rubriques@gmail.com).

La souplesse temporelle du client est de plus en plus sollicitée par les prestataires de service. Elle fait l'objet de transactions qui peuvent s'analyser comme de véritables coopérations temporelles. Quatre formes de souplesse temporelle sont identifiées et leurs enjeux analysés. Les exemples montrent les pratiques actuelles, traditionnelles ou innovantes. Demain, davantage de services pourraient utiliser ces précieux leviers.

Mołs-clés : coopération, négociation, valeur, temps, flexibilité.
THE MOBILISATION OF THE TEMPORAL FLEXIBILITY OF THE CUSTOMER, AN EXPANDING STRATEGY IN SERVICE ACTIVITIES

The temporal flexibility of the customer is increasingly sought by service providers. The temporal transactions conducted with customers can be regarded as a genuine temporal cooperation. Four types of temporal flexibility are identified and their issues considered. Some examples illustrate the current practices, traditional or innovative. Tomorrow, more service activities could use these valuable levers. Key Words: cooperation, negotiation, value, time, flexibility. 
$\mathrm{D}$ es parcs de loisirs ouvrent davantage de spectacles nocturnes pour attirer une clientèle du soir (ex : Parc du Puy du fou). Des hôtels commencent à proposer des offres "flexibles" ou "non flexibles" à prix différenciés (ex : Hôtel La Pérouse à Nice). De plus en plus ostensiblement, les services qui accueillent du public cherchent à influencer le client dans la gestion de son temps, déployant une variété d'offres et d'options, visant à mobiliser sa souplesse temporelle.

Dans un contexte général d'accélération de l'action, observé par les sociologues (1), le temps est plus que jamais une ressource essentielle pour les entreprises et leurs clients. Demain, verrons-nous les cinémas, les magasins ou les cliniques fonctionner sur les mêmes principes ? Cet article propose de mettre en lumière le phénomène, d'en dégager les enjeux, d'observer les pratiques actuelles des entreprises pour ensuite formuler des recommandations et conclure sur un plan théorique.

\section{Les formes négociables de souplesse temporelle}

La souplesse temporelle du client intéresse depuis longtemps les prestataires de service. Elle peut se définir comme la tendance du client à modifier ses plans d'organisation, en fonction des circonstances. Par exemple, un client qui voulait partir un week-end en Provence en juin se rend compte que les prix sont très attractifs en avril, ce qui l'amène à anticiper son projet. La mobilisation de cette souplesse par le prestataire, afin d'attirer le client vers certaines plages de temps, peut prendre plusieurs formes $(4,7,11)$.

La première forme de mobilisation est relative au moment de l'usage du service. Elle consiste à offrir des avantages aux clients qui acceptent de venir en période creuse. Par exemple : des hôtels pratiquent des prix réduits le week-end, des centres de thalassothérapie offrent des soins en plus hors saison. Cette forme peut s'appeler souplesse période creuse (forme 1).

Une deuxième forme concerne le temps de l'achat. Le client qui achète tard peut parfois obtenir des "soldes". Dans le secteur du voyage, cette pratique s'est institutionnalisée sous le nom de VDM (vente de dernière minute). Des sites Internet spécialisés offrent des bonnes affaires aux clients prêts à partir rapidement. Cette forme peut s'appeler souplesse achat tardif (forme 2).

La souplesse achat précoce (forme3) fonctionne pratiquement en sens inverse. Plus le client achète à l'avance, plus il a des chances d'obtenir des prix bas.Tout type de service à réservation peut utiliser cet "early booking", mais cette pratique est souvent liée au yield management (noté par la suite YM) et à ses quotas de prix bas, moyens et élevés (5). Inventé à la fin des années soixante-dix par le secteur aérien, le YM est aujourd'hui considéré comme très efficace. Il a fait tache d'huile dans le secteur ferroviaire et continue à s'étendre dans l'hôtellerie et dans d'autres services à réservation.

La quatrième forme est en train d'évoluer rapidement sous nos yeux. Elle ne concerne plus le moment où le client agit (usage ou achat), mais la flexibilité de son temps entre le moment de la réservation et celui de l'usage. Dans sa variante la plus courante, l'acheteur renonce à son droit traditionnel et implicite de pouvoir modifier sa réservation. Il réserve "ferme» et fait en quelque sorte un pari sur le futur en vue d'obtenir un prix bas. Cette forme peut s'appeler souplesse de modification ou encore flexibilité, selon un mot qui émerge actuellement des offres (forme 4).

\section{Les enjeux de la mobilisation temporelle}

Si la souplesse temporelle du client est tant sollicitée, c'est que prestataires et clients y trouvent avantage. Ces arrangements précis et subtils, véritables formes de coopérations temporelles, sont facilités par les technologies de l'information et de la communication (notées par la suite TIC).

Les prestataires qui accueillent du public ont toujours des moyens d'accueil limités (mètres carrés, personnel). La bonne utilisation de ces moyens, synonyme de " remplissage " élevé et stable au fil du temps, est un déterminant essentiel de la rentabilité. Mais cette bonne utilisation est difficile à obtenir, car la demande est par nature fluctuante. Pour résoudre ce problème reconnu comme général dans les services, deux politiques sont classiquement préconisées $(7,11)$ : s'adapter à la demande (ex : fermer certains jours) et/ou "gérer la demande" en la lissant, ce qui revient à jouer avec la souplesse temporelle des clients. Les enjeux du lissage sont très forts. Les hôteliers de station de ski disent que s'ils ratent une semaine d'activité, ils ratent toute la saison ; les compagnies aériennes disent que leur rentabilité dépend d'une bataille qui se joue sur chaque siège de chaque avion. Du coup, les facilités qu'il semblait naturel d'offrir au client il y a peu - le client vient quand il veut, il a le droit de changer d'avis - sont de plus en plus remises en question à mesure que les techniques de négociation s'affinent.

Le client, quant à lui, bénéficie d'avantages. Mais, il doit accepter les périodes les moins demandées et avoir un comportement planificateur ou, au contraire, accepter le risque de ne pas obtenir exactement ce qu'il souhaite pour les VDM. Cela implique un certain travail de sa part (3). Il doit être en veille pour obtenir les bonnes informations. Certains n'hésitent pas à passer des heures à l'affût pour comparer les offres ou pour sauter sur les bons plans dès qu'ils apparaissent sur Internet. Par contre, d'autres clients n'ont pas envie d'assumer de 
telles contraintes, ou n'ont pas la capacité de naviguer sur le Web. D'autres encore disposent de peu de souplesse temporelle, en particulier lorsqu'ils ont de jeunes enfants (calendrier scolaire). Cette inégalité incontestable, qui oblige certains clients à payer plus cher pour la même chose, est parfois dénoncée ouvertement (3).

Ces évolutions seraient impossibles sans les progrès des TIC. Ces technologies permettent maintenant de gérer quasi-automatiquement des milliers de capacités et de les offrir, facilement et en temps réel, aux yeux du monde entier. Rappelons qu'un seul hôtel de 100 chambres représente 36500 réservations dans l'année, sans compter les modifications. Du coup, les clients tâtonnent pour trouver les meilleures solutions. (voir encadré 1). Les prestataires modifient leurs offres, en fonction des réactions des clients. Une véritable " négociation implicite " se met en place, pour reprendre un terme employé par Prahalad et Ramaswamy (10). «Nous entrons dans un monde où la valeur résulte d'une négociation implicite entre le consommateur et l'entreprise» (p3, notre traduction). Au temps du fax, quand il fallait trois jours pour confirmer un voyage, la souplesse temporelle du client n'était guère mobilisable.

Ce nouveau type de négociation permet de connecter les ressources temporelles des deux parties. Pour tenter de mieux définir le phénomène, nous proposons de l'inscrire dans le cadre général de la "co-création de valeur". Ce cadre convient dès lors qu'entreprises et clients s'apportent mutuellement de la valeur et mobilisent leurs ressources respectives. Il donne lieu actuellement à un intense courant de recherche, suite à des contributions majeures $(9,10,12,13)$ et englobe de nombreuses réalités, telles que la co-innovation ou la co-promotion (2).A côté des formes les plus étudiées de co-création, comme lorsque le client coproduit pour lui-même (self-service) ou directement pour l'entreprise (conception de publicités), la mobilisation de la souplesse temporelle apparaît comme une forme assez particulière et plus indirecte. Elle correspond à une véritable coopération temporelle, une synchronisation entre le temps du producteur et celui du client, qui améliore l'efficacité économique globale par une meilleure utilisation des moyens productifs, et ce, même si le client opportuniste a peu conscience de sa contribution. Cette coopération est intimement liée aux stratégies marketing, à la présentation des offres et à la fixation des prix.

\section{La mobilisation temporelle en pratique}

Trois exemples peuvent illustrer comment les entreprises mobilisent en pratique la souplesse du client.

Les parcs de loisirs français (Disney, Puy-du-Fou, Futuroscope etc.) affichent actuellement de bons résultats. Selon certains observateurs (8) ceci est dûu, en plus du renouvellement systématique des manèges et des attractions, à différentes actions qui convergent pour attirer les clients sur certaines plages de temps. Cet exemple montre comment un service qui n'utilise pas le YM peut cependant se servir des quatre formes identifiées de souplesse temporelle (voir encadré 2).

Si le secteur du transport aérien utilise depuis longtemps les offres «à restriction» (modification ou annulation bloquées ou payantes), l'hôtellerie restait jusqu'ici assez fidèle à sa tradition généreuse : réservation souvent sans paiement, modifications et annulations presque gratuites. Mais, on commence à observer sur

\section{Encadré 1 : négociation implicite et intermédiaires du Web}

Exemple de navigation. Le 13 mai 2011 un consommateur envisage un week-end de 2 jours à Prague. II souhaite partir le vendredi soir du 17 juin et rentrer le dimanche soir.

- Etape 1 sur Google. L'internaute tape "Vol Paris Prague ". Aussitôt, toutes sortes de mots attrayants apparaissent à

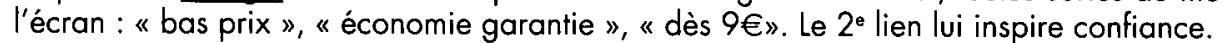

- Etape 2 sur Air France. L'offre est à $222 €$ avec de bons horaires. Mais sans le demander, les meilleurs prix des jours connexes apparaissent. Il saute aux yeux que le prix tombe à $100 €$ en décalant d'un jour. Altractif ! Mais le détail montre qu'il ne resterait plus qu'un seul jour de tourisme sur place, au lieu de deux (vol tard le samedi et tôt le lundi). Déception!

- Etape 3 sur Alibabuy. L'internaute passe alors sur un comparateur de prix. En un seul clic, il reçoit les meilleures offres de différentes compagnies, de $131 €$ à $216 €$. Mais, sans le demander, tout d'un coup, il est redirigé vers un site marchand.

- Etape 3 sur le site Opodo. L'internaute peut « filtrer les horaires », ce qui est très pratique. Pour son projet d'origine, avec les horaires souhaités, il reçoit une offre à $195 €$ (Smart Wings). S'il décale d'un jour en prenant son lundi de congé, le prix tombe à $154 €$ (Czech Airline). Cette solution lui convient très bien, car il aura 2 jours $1 / 2$ sur place pour les mêmes 2 nuits d'hôtel. Mais il hésite encore car il ne connaît pas cette compagnie.

- Etape 4. II revient dans Google et tape «avis de consommateur Czech Airline».

- Etape 5. Le site Ciao répond et présente 4 avis qui sont tous bons.

- Etape 6. Le lendemain, l'internaute revient sur Opodo pour réserver ferme.

- Au final, en 6 étapes et via 4 intermédiaires différents (soulignés), l'internaute a changé un peu son projet mais trouvé un bon prix (-30\%). Il se sent assez fier de ce résultat et de toujours mieux se débrouiller sur la toile. 


\section{Encadré 2 : Les parcs de loisirs}

Les parcs de loisirs accueillent le public sans tradition de réservation (sauf pour les groupes). Proposant des loisirs en extérieur, ils sont tributaires de la durée du jour et de la météo et ferment souvent dans la période d'hiver. Plusieurs de leurs actions convergent actuellement pour attirer le client là où il ne viendrait pas naturellement, ce qui leur assure une meilleure utilisation des moyens productifs.

Souplesse période creuse. Les parcs arrivent à valoriser certaines périodes autrefois délaissées : les spectacles nocturnes accroissent l'usage du parc sur la journée, tandis que des animations d'hiver permettent d'accroître cet usage sur l'année (ex : Féeries de Noël).

Souplesse achat tardif. Lorsque les prévisions d'activité sont faibles, les parcs activent des promotions de dernière minute offertes uniquement à une population de proximité (diffusions de coupons dans les boîtes aux lettres). Cette population se montre très sensible aux faveurs accordées et réagit positivement à ces promotions.

Souplesse achat précoce. Les réservations s'ouvrent à un public BtoC et sont encouragées par des offres spéciales sur Internet. Du coup, les clients qui réservent à l'avance peuvent être orientés sur les périodes qui intéressent le prestataire. L'achat précoce présente aussi d'autres avantages pour le prestataire : financiers (si le service est payé d'avance) et concurrentiels (les offres concurrentes ne sont plus vues, même si entre temps elles s'avèrent mieux placées).

Souplesse de modification. Les entrées réservées à l'avance sont liées à plus ou moins de flexibilité en termes de modification ou d'annulation.

Enseignements stratégiques. Cet exemple, en forme de success story, montre que les prestataires peuvent animer les périodes creuses sans les considérer comme une fatalité. Les soldes de dernière minute peuvent s'activer en diffusion ciblée pour éviter une perte d'image et une trop forte baisse du ticket moyen. La réservation peut s'activer ou s'élargir, pour permettre la « négociation implicite».

Internet un phénomène assez nouveau. Certains hôtels proposent explicitement des chambres flexibles ou non flexibles (ex : hôtel La Pérouse à Nice, chambre à $215 €$ en "flexible annulable gratuitement jusqu'à J-2 ", et à $180 €$ en "flash non modifiable», chiffres relevés en mars 2011 pour une chambre un mois plus tard). Ceci suggère deux réflexions. Les pratiques des secteurs en pointe pour la négociation temporelle continuent à se répandre à d'autres secteurs. L'idée que la flexibilité du client est une valeur ouvertement négociable fait son chemin.

Dans la même veine, nous avons observé en Suède un prestataire de transport ferroviaire qui innove en la matière (compagnie SJ). Il ne propose plus d'offres non flexibles moins chères, noyées dans des forfaits peu transparents et à géométrie variable (pour le train en France le client doit se repérer entre le tarif Normal, Loisir, Loisir réduit, Prem's et Pro). Il propose au client de choisir d'abord son trajet, puis ses options de flexibilité (ex : trajet à $40 €$, droit d'annulation à $4 €$, droit de modification à $1 €$ ). Ce type d'offre est attractif pour le client, sans brider le prestataire. L'affichage est simple et clair, le choix est positif et responsable. A l'instar du prestataire, qui s'engage ferme lors de la réservation, le client s'engage ferme également, mais il peut s'il le veut acheter une valeur supplémentaire payante : la flexibilité. Il construit ainsi lui-même sa propre valeur d'usage, composée ici de deux éléments distincts : le service d'une part, la flexibilité d'autre part.

\section{Recommandations}

Les observations précédentes permettent d'avancer quelques recommandations, fondées sur ces coopéra- tions temporelles. Adressées à tout service accueillant du public, ces recommandations diffèrent selon que le service utilise la réservation (avec YM ou sans), ne l'utilise pas du tout ou l'utilise partiellement.

Pour les services «à réservation " qui pratiquent le YM, les conseils seraient de :

- Proposer plus systématiquement des réservations non flexibles assorties d'options de flexibilité. La possibilité de modifier et d'annuler induit des coûts pour l'entreprise et correspond à une valeur pour le client, détachable et monnayable. Ex : trains suédois.

- Être plus transparent sur les réductions possibles et leurs conditions. Alors que le YM a acquis une réputation d'opacité, une présentation différente de l'information, dans un esprit plus coopératif, permettrait aux entreprises d'être encore plus efficaces et d'améliorer leurs relations avec les clients.

Pour les services «à réservation " qui ne pratiquent pas le YM.

- Il est possible de traiter moins généreusement qu'aujourd'hui les clients qui coopèrent mal. Ex : les services de santé pourraient pénaliser les clients qui honorent mal leurs rendez-vous, voire les facturer.

Pour les services qui ne pratiquent pas du tout la réservation, les leviers d'actions sont plus réduits mais existent cependant.

- Elargir les périodes d'ouverture en offrant des produits enrichis ou différents. Ex : les parcs de loisirs.

- Utiliser les variations de prix pour dynamiser les heures creuses, ce qui est imaginable même sans tradition dans ce domaine (voir encadré 3). 


\section{Encadré 3 : Fixer les prix différemment dans le commerce?}

Chaque secteur possède ses traditions, y compris pour fixer les prix. Imaginons qu'une enseigne souhaite mieux lisser son activité. Elle pourrait, en le faisant largement savoir :

- Accorder des remises après passage en caisse en fonction de l'affluence. Imaginons : - 5\% la première heure d'ouverture chaque jour, $+5 \%$ le vendredi soir. Les grandes enseignes avancent d'ailleurs déjà dans cette voie, mais de manière limitée. Ex : Chez Carrefour, les porteurs de la carte Pass bénéficient de $5 \%$ de réduction par tranche de $60 €$ le mardi (ou un autre jour creux selon les magasins).

- Faire varier les prix étiquetés. Dans le même esprit mais à plus grande échelle, les étiquettes électroniques rendent possible toutes sortes de variations, légales tant que l'affichage reste clair pour le client.

- Faire payer un droit d'entrée, en modifiant complètement les pratiques. Imaginons : $1 €$ la demi-heure en semaine, $2 €$ le week-end, et gratuit à certaines heures. Des distributeurs «low cost» pourraient être intéressés par de telles formules, qui de plus auraient l'avantage de dissuader les clients «ventouse» (ceux qui viennent davantage pour se distraire que pour acheter).

Ces tarifs seraient-ils plus choquants que ceux pratiqués dans l'aérien ? Le fait est qu'ils reposeraient sur des logiques similaires.

Pour les services qui pratiquent peu la réservation.

- Elargir cet usage de la réservation. Les avantages sont nombreux car, en plus d'ouvrir la voie à la négociation implicite, la réservation rend le client moins anonyme et permet d'instaurer des relations plus durables. Ex : Certains cinémas remplacent leurs caisses traditionnelles par des bornes automatiques, tout en instaurant la réservation Internet. Les spectateurs peuvent alors être tentés par des jours ou des heures qu'ils n'auraient pas choisis spontanément. Par la suite, ils bénéficieront d'informations et d'offres personnalisées.

\section{Conclusion}

Dans cet article, nous avons souhaité jeter un éclairage sur les pratiques actuelles des entreprises de service qui sollicitent la souplesse temporelle de leurs clients. Nous avons vu pourquoi cette souplesse temporelle constitue une véritable ressource, et comment elle est mobilisée. Mais ces pratiques sont-elles vraiment nouvelles ? Ne sont-elles pas identifiées depuis longtemps?

Certes, les pratiques détaillées dans l'article sont déjà connues, mais le contexte Internet change radicalement la donne. Il intensifie, élargit et affine l'usage de ces pratiques. L'information se diffuse rapidement, elle est traitée en temps réel, elle circule avec fluidité dans les deux sens. Le consommateur plus actif cherche les «bons plans», il est prêt à s'adapter en fonction des offres et à rentrer dans cette négociation implicite à grande échelle. Des pratiques utilisées jusqu'ici surtout par le YM se diffusent à d'autres services, de nouvelles formes de flexibilité apparaissent tandis que la pratique de la réservation s'élargit. A l'avenir, la diffusion de l'Internet Mobile (Smartphones) va encore intensifier cette dynamique, qu'il est fondamental de comprendre et d'utiliser stratégiquement.

Du coup, cette nouvelle réalité rejaillit au plan théorique. Nous proposons de faire un point sur le thème de "la gestion de la demande ", en adoptant une perspective historique qui, dans notre vision, intègre deux sauts technologiques d'importance :l'informatique BtoB, puis l'Internet.

- Dans les années 70, Sasser (11) explique déjà comment dynamiser les périodes creuses, par des actions diverses proches de notre forme 1 (prix en moins, produit en plus ou différent). Dans son article, la forme 2 (soldes) est passée sous silence car sans doute considérée comme peu recommandable. Quant à la réservation, elle est évoquée, mais comme simple moyen d'étaler les rendez-vous, sans idée de moduler les prix.

- Dans les années 80-90, l'informatique BtoB permet le développement du YM qui, pour éviter le bradage systématique des formes 1 et 2 (période creuse et soldes), donne corps aux formes 3 et 4 (achat précoce et flexibilité). Mais il est intéressant de remarquer que ces formes sont conçues comme des "restrictions " pour limiter l'accès aux prix bas à certains segments. Cette logique de segmentation est très claire dans la littérature, quand le YM se définit comme une technique permettant de vendre "la bonne capacité " au "bon type de client ", au "bon moment ", et avec le "bon prix" (6). Autrement dit, l'entreprise conçoit le YM comme un stratagème permettant de "trier" les clients, et non comme une technique de négociation. Cette segmentation est facilitée par des intermédiaires, qui ont seuls accès à l'information et proposent des prix élevés aux segments aisés et aux entreprises.

- Dans les années 2000-2010, Internet rend l'information accessible à un public très large. Les clients s'initient au tâtonnement autour de différentes solutions, aidés d'abord par de puissants moteurs de recherche, puis de nos jours par les comparateurs de prix (encadré 1). Les clients se laissent moins " trier " et les hommes d'affaires découvrent les prix bas. Dès lors, il nous semble que la logique de segmentation, pilotée d'en haut par l'entreprise, cède le pas à une autre logique, plus équilibrée, de négociation et de coopération. 


\section{Mobiliser la souplesse temporelle du client}

\section{Références}

(1) Aubert N. (2003), Le culte de l'urgence. La société malade du temps, Coll. Champs essais, Paris, Flammarion.

(2) Cova B. (2008), Consumer made, quand le consommateur devient producteur, Décisions Marketing, 50, avril, 1927.

(3) Dujarier M.A. (2008), Le travail du consommateur. De $M c D o$ à eBay : comment nous coproduisons ce que nous achetons, Paris, La Découverte.

(4) Durrande-Moreau (2002), Services et tactiques de prix, quelles spécificités ?, Décisions Marketing, 25, janviermars, 17-28.

(5) Guilloux V. (2000), Le Yield en marketing : concepts, méthodes et enjeux stratégiques, Recherche et Applications en Marketing, 15, 3, 55-73.

(6) Kimes S.E. (1989), Yield Management: A Tool for Capacity-Constrained Service Firms, Journal of Operations Management, 8, 4, 348-363.
(7) Lovelock C. and Wirtz J. (2007), Services marketing, people, technology, strategy, 6è éd., Pearson International Edition.

(8) Manceau J.J. (2008), Le nouveau souffle des parcs de loisirs, L'Expansion, 735, 116-117.

(9) Normann R. (2001), Reframing Business: When the Map Changes the Landscape, New-York, John Wiley \& sons.

(10) Prahalad C.K. and Ramaswamy V. (2004), Co-creation experiences: the next practice in value creation, Journal of Interactive Marketing, 18, 3, 1-10.

(11) Sasser W.E. (1976), Match supply and demand in service industries, Harvard Business Review, 54, 6, 133-140.

(12) Stabell C.B. and Fjeldstad O.D. (1998), Configuring value for competitive advantage: on chains, shops, and networks, Strategic Management Journal, 19, 5, 413-437.

(13) Vargo S. and Lusch R. (2004), Evolving to a new dominant logic for marketing, Journal of Marketing, 68, 1, 1-17. 
Copyright of Decisions Marketing is the property of AFM c/o ESCP-EAP and its content may not be copied or emailed to multiple sites or posted to a listserv without the copyright holder's express written permission.

However, users may print, download, or email articles for individual use. 